\title{
Fluctuating local moments, itinerant electrons, and the magnetocaloric effect: Compositional hypersensitivity of FeRh
}

\author{
J. B. Staunton, ${ }^{1, *}$ R. Banerjee, ${ }^{1}$ M. dos Santos Dias, ${ }^{2}$ A. Deak, ${ }^{3}$ and L. Szunyogh ${ }^{4}$ \\ ${ }^{1}$ Department of Physics, University of Warwick, Coventry CV4 7AL, United Kingdom \\ ${ }^{2}$ Peter Grünberg Institut and Institute for Advanced Simulation, Forschungszentrum Jülich and JARA, D-52425 Jülich, Germany \\ ${ }^{3}$ Department of Theoretical Physics, Budapest University of Technology and Economics, Budapest, Hungary \\ ${ }^{4}$ Condensed Matter Research Group of the Hungarian Academy of Sciences, Budapest University of Technology and Economics, \\ Budapest, Hungary \\ (Received 3 January 2014; revised manuscript received 10 February 2014; published 25 February 2014)
}

\begin{abstract}
We describe an ab initio disordered local moment theory for materials with quenched static compositional disorder traversing first-order magnetic phase transitions. It accounts quantitatively for metamagnetic changes and the magnetocaloric effect. For perfect stoichiometric B2-ordered FeRh, we calculate the transition temperature of the ferromagnetic-antiferromagnetic transition to be $T_{t}=495 \mathrm{~K}$ and a maximum isothermal entropy change in $2 \mathrm{~T}$ of $|\Delta S|=21.1 \mathrm{~J} \mathrm{~K}^{-1} \mathrm{~kg}^{-1}$. A large (40\%) component of $|\Delta S|$ is electronic. The transition results from a fine balance of competing electronic effects which is disturbed by small compositional changes; e.g., swapping just $2 \%$ Fe of "defects" onto the Rh sublattice makes $T_{t}$ drop by $290 \mathrm{~K}$. This hypersensitivity explains the narrow compositional range of the transition and impurity doping effects.
\end{abstract}

DOI: 10.1103/PhysRevB.89.054427

\section{INTRODUCTION}

When a metal goes through a change of magnetic order, the complex electronic fluid with its emergent magnetic fluctuations transforms. The magnetic effect on structure, electronic transport, and so on is particularly dramatic at first-order phase transitions, and modest changes in composition and quenched disorder are strongly influential, shifting, broadening [1,2], or removing transitions entirely. In a metallic material the changes to the electronic structure as it passes through a first-order magnetic transition can be significant. In this paper we show how these changes can be determined, find out how they are affected by composition and disorder, and trace their impact back on the transition itself. We find a particularly striking example in the metamagnetic Fe-Rh material [3-10].

In roughly equal proportions, iron and rhodium order into a $\mathrm{B} 2(\mathrm{CsCl})$ alloy phase which experiences a first-order ferromagnetic $(\mathrm{F})$ to antiferromagnetic $(\mathrm{AF})$ phase transition at $T_{t}$ around $340 \mathrm{~K}$, below a $T_{c}$ of $670 \mathrm{~K}$. This property is highly composition-dependent so that the F-AF transition vanishes in alloys with as little as a $2 \%$ iron excess or deficiency and $T_{t}$ varies strongly with sample preparation, irradiation, and addition of impurities [11-17]. For example a little $\mathrm{Pd}$ raises $T_{t}$ while doping with $\mathrm{Pt}$ suppresses it. The $1 \% \mathrm{Fe}$-deficient alloy shows one of the largest recorded magnetocaloric effects around $T_{t}[4,18]$ which deteriorates on subsequent magnetic and thermal cycling. The prominent F-AF transition is also relevant for the design of ultrahigh density magnetic recording media as shown by FePt/FeRh bilayer investigations [19] and its time dependence has also been probed by a recent suite of experiments and analysis [20-23].

The electronic source of the F-AF transition has been tracked down carefully by several spin-density-functional theory (SDFT) based studies [21,23-26], which have also

\footnotetext{
*j.b.staunton@warwick.ac.uk
}

PACS number(s): 75.10.Lp, 75.30.Kz, 75.30.Sg, 75.47.Np

looked at the role of spin waves [27], and much insight has been gained. For an AF state with zero sum magnetization on the Fe sites, the Rh related states have no net spin polarization and there is a rough half filling of some of the Fe-related $d$ bands, which sustains the long-range AF order [28]. Around the Fermi energy $E_{\mathrm{F}}$, there is a strong hybridization between $\mathrm{Fe}$ and $\mathrm{Rh}$ states in both spin channels for both $\mathrm{F}$ or AF order. In the former case, however, where the Rh sites pick up an overall spin polarization $[25,26]$, some bonding states are pulled down in energy enhancing the F state. The balance between these two competing effects drives the F-AF transition. We show in this paper, by allowing for finite $T$ magnetic fluctuation effects, that tiny alterations of composition and quenched disorder change the electronic structure to tip this balance which makes $T_{t}$ and the critical fields $H_{c}$ triggering the transition highly composition sensitive.

Long-range compositional order is never perfect in any real Fe-Rh sample and there can also be a slight off-stoichiometry. Given that the alloy orders from a $\mathrm{Fe}_{50} \mathrm{Rh}_{50}$ solid solution around $1600 \mathrm{~K}$ [5], a simple Bragg-Williams model [29] analysis indicates that at least $1 \%$ or $2 \%$ of the sites on the $\mathrm{Rh}$ cubic sublattice will be occupied by $\mathrm{Fe}$ and vice versa following typical annealing and cooling processes. The number of Fe-occupied $\mathrm{Rh}$ sublattice sites diminishes for marginally Fe-poor alloys. Our theoretical work finds the F-AF transition to be profoundly influenced by such Fe antisite defects and explains the narrow range of composition for the transition. Added impurities affect the transition by how easily they displace $\mathrm{Fe}$ atoms onto such defects.

In the next section we describe our ab initio theory for metamagnetic transitions and the magnetocaloric effect which we then go on to apply to FeRh in the following section. We show how the theory can be applied to materials with quenched static compositional disorder and examine effects of this on this alloy system's first-order metamagnetic transition. We summarize, draw some overall conclusions, and assess the potential of the theory for aiding magnetic materials design in the last section. 


\section{AB INITIO THEORY OF METAMAGNETIC TRANSITIONS AND THE MAGNETOCALORIC EFFECT}

\section{A. Disordered local moments and the free energy}

Important magnetic fluctuations in a metal can often be modelled as "local moments," a picture captured by a generalization of SDFT [30] for noncollinear spin polarization. A separation of time scales between fast and slow electronic degrees of freedom causes local moments with slowly varying orientations $\left\{\hat{e}_{i}\right\}$ to emerge from the interacting electron system [31-37]. This means that "disordered local moments" (DLMs) are sustained by and influence the faster electronic motions. Their interactions with each other depend on the type and extent of the long-range magnetic order through the associated spin-polarized electronic structure [38] which itself adapts to the extent of magnetic order. Ensemble averages over all the appropriately weighted noncollinear local moment orientational configurations $\left\{\hat{e}_{i}\right\}$ are required for a realistic evaluation of the system's magnetic properties [34,39]. Here we develop the DLM theory for a magnetic material in an external magnetic field $\vec{H}$ at a temperature $T$ for application to systems with quenched disorder like our FeRh case study. We show how entropy changes that occur at magnetic transitions, the magnetocaloric effect (MCE), can be calculated ab initio, and quantify the potentially significant electronic contribution and its signature in temperature-dependent magnetotransport properties [10].

For an Fe-Rh alloy close to equiatomic stoichiometry and nearly complete B2-type order there are two atomic sites per unit cell in the cubic crystal lattice. One sublattice $(A)$ has sites, labeled $a$, largely Fe occupied but with a small percentage $(x)$ of sites occupied by $\mathrm{Rh}$ atoms. The other sublattice $(B)$, with sites $b$, mostly occupied by Rh atoms, has a small fraction $(y)$ $\mathrm{Fe}$ occupied. The alloy is designated $\mathrm{Fe}_{1-x} \mathrm{Rh}_{x}-\mathrm{Rh}_{1-y} \mathrm{Fe}_{y}$. A particular distribution of $\mathrm{Fe}$ and $\mathrm{Rh}$ atoms over the two sublattices is specified with $\left(\left\{\xi_{a}\right\},\left\{\xi_{b}\right\}\right)$, where $\xi_{a(b)}=\{0,1\}$ means site $a(b)$ is $\{\mathrm{Rh}, \mathrm{Fe}\}$ occupied, such that $\left\langle\xi_{a}\right\rangle=c_{A}=$ $1-x$, and likewise $\left\langle\xi_{b}\right\rangle=c_{B}=y$. The probability that the system's local moments are configured according to $\left\{\hat{e}_{a}\right\},\left\{\hat{e}_{b}\right\}$ is

$$
P\left(\left\{\hat{e}_{a}\right\},\left\{\hat{e}_{b}\right\}\right)=\frac{\exp \left[-\beta \Omega\left(\left\{\hat{e}_{a}\right\},\left\{\hat{e}_{b}\right\}\right)\right]}{\mathcal{Z}},
$$

where the partition function is

$$
\mathcal{Z}=\prod_{a} \int d \hat{e}_{a} \prod_{b} \int d \hat{e}_{b} \exp \left[-\beta \Omega\left(\left\{\hat{e}_{a}\right\},\left\{\hat{e}_{b}\right\}\right)\right],
$$

$1 / \beta=k_{\mathrm{B}} T$, and the free energy

$$
\mathcal{F}=-k_{\mathrm{B}} T \ln \mathcal{Z}
$$

A “generalized" electronic grand potential $\Omega\left(\left\{\hat{e}_{a}\right\},\left\{\hat{e}_{b}\right\}\right.$; $\left.\vec{H},\left\{\xi_{a}\right\},\left\{\xi_{b}\right\}, T\right)$ is in principle available from SDFT [30] where, for fixed $\vec{H}$ and for the arrangement of atoms specified by $\left\{\xi_{a}\right\}$ and $\left\{\xi_{b}\right\}$, the spin density is constrained to comply with the local moment configurations $\left\{\hat{e}_{a}\right\}$ and $\left\{\hat{e}_{b}\right\}$. It thus plays the role of a local moment Hamiltonian but its electronic glue origins can make it complicated.
Expanding about a suitable reference "spin" Hamiltonian [40],

$$
\Omega_{0}=\sum_{a} \vec{h}_{a} \cdot \hat{e}_{a}+\sum_{b} \vec{h}_{b} \cdot \hat{e}_{b}
$$

gives a mean-field theoretical estimate of the free energy [30]. A similar single-site approximation averages over atomic configurations with the assumption that atomic diffusion times are very long and that the composition is fixed by the material's preparation. Local moments establish on the Fe atoms only and, in line with $T=0 \mathrm{~K}$ DFT studies [21] and consistent with other theoretical studies [25,26], a net spin polarization develops on the $\mathrm{Rh}$ atoms if there is an overall lining up of the Fe local moments, i.e., when $F$ order is established. The free energy is given by

$$
\begin{aligned}
\mathcal{F}=\bar{\Omega} & +c_{A} \sum_{a}\left(\mu_{a} \vec{m}_{a} \cdot \vec{H}-\frac{1}{\beta} \int \mathrm{d} \hat{e} P_{a}(\hat{e}) \ln P_{a}(\hat{e})\right) \\
& +c_{B} \sum_{b}\left(\mu_{b} \vec{m}_{b} \cdot \vec{H}-\frac{1}{\beta} \int \mathrm{d} \hat{e} P_{b}(\hat{e}) \ln P_{b}(\hat{e})\right)
\end{aligned}
$$

The free energy therefore comprises an internal energy $\bar{\Omega}$ from the interacting electron system averaged over local moment and compositional configurations, and two extra contributions from the Fe local moments in each sublattice: the interaction with the external magnetic field $\vec{H}$ and the magnetic entropy, $-T \bar{S}_{\text {mag }}$. The magnitudes of the local moment on each site, $\mu_{a}$ and $\mu_{b}$, are determined by the generalized SDFT. $\bar{\Omega}$ includes the effect on the spin-polarized electron density at the Rh sites from $\vec{H}$ and the magnetic order of the Fe local moments $[41,42]$. The probability of an Fe local moment being oriented along $\hat{e}_{a}$ on site $a$ of the Fe-rich sublattice is set as

$$
P_{a}\left(\hat{e}_{a}\right)=\frac{\exp \left[\vec{\lambda}_{a} \cdot \hat{e}_{a}\right]}{\int d \hat{e}_{a} \exp \left[\vec{\lambda}_{a} \cdot \hat{e}_{a}\right]}
$$

and similarly for an Fe atom defect on the Rh-rich sublattice, $P_{b}\left(\hat{e}_{b}\right)$. A magnetic state is specified by the set of local order parameters,

$$
\begin{gathered}
\left\{\vec{m}_{a}=\int d \hat{e}_{a} P_{a}\left(\hat{e}_{a}\right) \hat{e}_{a}=\left[\operatorname{coth}\left(\lambda_{a}\right)-1 / \lambda_{a}\right] \hat{\lambda}_{a}\right\} \\
\left\{\vec{m}_{b}=\int d \hat{e}_{b} P_{b}\left(\hat{e}_{b}\right) \hat{e}_{b}=\left[\operatorname{coth}\left(\lambda_{b}\right)-1 / \lambda_{b}\right] \hat{\lambda}_{b}\right\},
\end{gathered}
$$

each of which can take values between 0 and 1 .

The parameters $\left\{\vec{\lambda}_{a}\right\},\left\{\vec{\lambda}_{b}\right\}$ are given as $\left\{\beta \vec{h}_{a}\right\},\left\{\beta \vec{h}_{b}\right\}$, respectively, where the Weiss fields satisfy

$$
\begin{aligned}
\vec{h}_{a} & =-\frac{1}{c_{A}} \frac{\partial \bar{\Omega}_{a}}{\partial \vec{m}_{a}}+\mu_{a} \vec{H}, \\
\vec{h}_{b} & =-\frac{1}{c_{B}} \frac{\partial \bar{\Omega}}{\partial \vec{m}_{b}}+\mu_{b} \vec{H} .
\end{aligned}
$$

This ensures that the function $\mathcal{F}\left(\left\{\vec{m}_{a}\right\},\left\{\vec{m}_{b}\right\} ; \vec{H}, c_{A}, c_{B}, T\right)$, shown in Eq. (5), is minimized with respect to the $\left\{\vec{\lambda}_{a}\right\}$, $\left\{\vec{\lambda}_{b}\right\}$ (equivalently $\left\{\vec{m}_{a}\right\},\left\{\vec{m}_{b}\right\}$ ), at a temperature $T$, and hence describes the free energy. 


\section{B. Entropy changes}

Particularly pertinent for our description of MCE [43] is the electronic entropy contained in $\bar{\Omega}$ of Eq. (5) [44],

$$
\bar{\Omega}=\bar{E}-T \bar{S}_{\text {elec }}
$$

$\bar{E}$ is the SDFT-based energy averaged over local moment orientations and compositional arrangements [38,39,45-47] with electronic density of states (DOS) at the Fermi energy $\bar{n}\left(E_{\mathrm{F}} ;\left\{\vec{m}_{a}\right\},\left\{\vec{m}_{b}\right\} ; \vec{H}, c_{A}, c_{B}\right)$ and $\bar{S}_{\text {elec }}$ is the electronic entropy. $\bar{S}_{\text {elec }} \approx \frac{\pi^{2}}{3} k_{B}^{2} T \bar{n}\left(E_{\mathrm{F}}\right)$ from the Sommerfeld expansion. The isothermal entropy difference between states with and without a magnetic field applied, $\Delta S(\vec{H}, T)$, is therefore comprised of the sum of the $\bar{S}_{\text {mag }}$ and $\bar{S}_{\text {elec }}$ differences. Likewise the adiabatic temperature change, $\Delta T_{a d}(\vec{H}, T)$ can be estimated from $\bar{S}_{\text {mag }}(\vec{H}, T)+\bar{S}_{\text {elec }}(\vec{H}, T)+\bar{S}_{\text {latt }}(T)=\bar{S}_{\text {mag }}\left(\overrightarrow{0}, T+\Delta T_{a d}\right)+$ $\bar{S}_{\text {elec }}\left(\overrightarrow{0}, T+\Delta T_{a d}\right)+\bar{S}_{\text {latt }}\left(T+\Delta T_{a d}\right)$ where $\bar{S}_{\text {latt }}$ is the lattice vibration entropy.

\section{Calculational details: The Weiss fields and $T$-dependent electronic structure}

We use a multiple-scattering [Korringa-Kohn-Rostoker (KKR)] [39,45-47] formalism to describe the electronic structure and the averaging over local moment configurations is carried out via the coherent potential approximation (CPA) $[45,47]$ technology. Relativistic spin-orbit coupling effects on the electronic motion are fully included by solving the Kohn-Sham-Dirac equations [48] and the maximum value of the orbital angular momentum used to describe the scattering is $l_{\max }=3$. The electronic charge density and also the magnetization density, which sets the magnitudes, $\mu_{a}, \mu_{b}$, of the local moments, are determined from a self-consistent field (SCF)-KKR-CPA [46] calculation using the muffintin approximation and the local spin-density approximation (Perdew-Wang [49]) for exchange and correlation. In this work we use potentials, charge, and magnetization densities generated self-consistently for the paramagnetic DLM state $\left(\left\{\vec{m}_{a}=0\right\},\left\{\vec{m}_{b}=0\right\}\right)$. Using them with the Harris expression [50] to calculate an energy for $\left\{\vec{m}_{a}=1\right\},\left\{\vec{m}_{b}=1\right\}$ and comparing it with the total energy of the $T=0 \mathrm{~K}$ FM state in which the charge and magnetization densities are found fully self-consistently, we account approximately for the changes to charge and magnetization densities that are induced for intermediate values of the magnetic order parameters $\left\{\vec{m}_{a}\right\}$ and $\left\{\vec{m}_{b}\right\}$. This amounts to a term $-\frac{1}{2} U\left(c_{A} M_{A}^{2}+c_{B} M_{B}^{2}\right)$ being added to $\bar{\Omega}$ where $\vec{M}_{A(B)}=\frac{1}{N_{A(B)}} \sum_{a(b)} \mu_{a(b)} \vec{m}_{a(b)}$ describes the average magnetization per site on sublattice $A(B)$. The added term contains the effect that the density surrounding a $\mathrm{Rh}$ site becomes spin polarized once there is an overall ferromagnetic alignment of the Fe local moments [41]. In a subsequent publication [42] we lift this approximation and show how full charge and magnetization self-consistency can be achieved in the DLM theory. We find both approaches to be in good agreement.

For a given set of potentials, electronic charge, and local moment magnitudes, the orientations of the local moments are accounted for by the similarity transformation of the single-site scattering $t$ matrices [39],

$$
\underline{t}_{a(b) ; F e}\left(\hat{e}_{a(b)}\right)=\underline{R}\left(\hat{e}_{a(b)}\right) \underline{t}_{a(b) ; F e}(\widehat{z}) \underline{R}\left(\hat{e}_{a(b)}\right)^{+},
$$

where for a given energy (not labeled explicitly) $\underline{t}_{a(b) ; F e}(\widehat{z})$ stands for the $t$ matrix with effective field pointing along the local $z$ axis [48] and $R\left(\hat{e}_{a(b)}\right)$ is a unitary representation of the $O(3)$ transformation that rotates the $z$ axis along $\widehat{e}_{a(b)}$. The underlining specifies a matrix in angular momentum and spin space.

The Weiss fields, $\vec{h}_{a}, \vec{h}_{b}$ [Eq. (8)], can be expressed in terms of the KKR-CPA formalism as follows as [39]

$$
\begin{aligned}
\vec{h}_{a(b)}= & U \mu_{a(b)} \vec{M}_{a(b)}+\mu_{a(b)} \vec{H}+\frac{3}{4 \pi} \int \hat{e}_{a(b)} \\
& \times\left[\int \frac{E_{\mathrm{F}}}{\operatorname{Im}} \ln \operatorname{det} \underline{D}_{a(b) ; F e}^{-1}\left(\varepsilon ; \hat{e}_{a(b)}\right) d \varepsilon\right] d \hat{e}_{a(b)},
\end{aligned}
$$

where

$$
\begin{aligned}
& \underline{D}_{a(b) ; F e}^{-1}\left(\varepsilon ; \hat{e}_{a(b)}\right) \\
& \quad=\left[\underline{1}+\left(\left[\underline{t}_{a(b) ; F e}\left(\hat{e}_{a(b)}\right)\right]^{-1}-\left(\underline{t}_{a(b)}\right)^{-1}\right) \underline{\bar{\tau}}_{a a(b b)}\right] .
\end{aligned}
$$

$\bar{t}_{a(b)}$ describes the CPA effective-medium single-site scatterer and $\underline{\bar{\tau}}_{a a(b b)}$ describes the CPA site-diagonal scattering path operator $[39,45,46]$ for a site $a(b)$ on the predominantly $\mathrm{Fe}$ (Rh) sublattice.

In this multiple-scattering KKR formalism the electronic structure setting and being set by the behavior of the fluctuating local moments is expressed in terms of the electronic Green function [47,51]. Its average over the local moment configurations appropriately weighted for a given temperature enables us to readily calculate temperature-dependent electronic effects. Work is in progress to incorporate these effects into a full Kubo-Greenwood-Středa formalism for $T$-dependent magnetotransport properties [52]. In the meantime we show here the $T$-dependent density of states as the first step to quantifying the magnetoresistive changes at $T_{t}$ as well as the electronic component of the MCE. For example, the density of states associated with sublattice $A$, partially averaged according to its occupation by $\mathrm{Fe}$ atoms, is given as

$$
\begin{aligned}
n_{A ; F e}(\varepsilon)= & -\frac{\operatorname{Im}}{\pi} \int d \hat{e}_{a} P_{a}\left(\hat{e}_{a}\right) \int d \mathbf{r}_{a} \\
& \times\left[\operatorname{Tr} \underline{G}_{\hat{e}_{a}, F e}\left(\mathbf{R}_{a}+\mathbf{r}_{a}, \mathbf{R}_{a}+\mathbf{r}_{a} ; \varepsilon\right)\right],
\end{aligned}
$$

where $\mathbf{R}_{a}$ is a lattice vector picking out a site on the $A$ sublattice, $\mathbf{r}_{a}$ is a position vector in the space around site $a$, and the Green function (all quantities have $\varepsilon$ dependence)

$$
\begin{aligned}
\underline{G}_{\hat{e}_{a}, F e} & \left(\mathbf{R}_{a}+\mathbf{r}_{a}, \mathbf{R}_{a}+\mathbf{r}_{a}\right) \\
= & \underline{Z}_{\hat{e}_{a}, F e}\left(\mathbf{r}_{a}\right) \underline{D}_{a ; F e}\left(\hat{e}_{a}\right) \underline{\tau}_{a a} \underline{Z}_{\hat{e}_{a}, F e}^{\dagger}\left(\mathbf{r}_{a}\right) \\
& \quad-\underline{Z}_{\hat{e}_{a}, F e}\left(\mathbf{r}_{a}\right) \underline{J}_{\hat{e}_{a}, F e}^{\dagger}\left(\mathbf{r}_{a}\right) .
\end{aligned}
$$

$\underline{Z}_{\hat{e}_{a}, F e}\left(\mathbf{r}_{a}\right)$ and $\underline{J}_{\hat{e}_{a}, F e}\left(\mathbf{r}_{a}\right)$ are the regular and irregular solutions of the Schrödinger (Dirac) equation [51] for a single site occupied by a Fe atom whose local moment is oriented along $\hat{e}_{a}$. The spin polarization of the DOS is given by replacing $\operatorname{Tr} \underline{G}_{\hat{e}_{a}, F e}\left(\mathbf{R}_{a}+\mathbf{r}_{a}, \mathbf{R}_{a}+\mathbf{r}_{a} ; \varepsilon\right)$ with $\operatorname{Tr} \underline{\sigma} \underline{G}_{\hat{e}_{a}, F e}\left(\mathbf{R}_{a}+\right.$ $\left.\mathbf{r}_{a}, \mathbf{R}_{a}+\mathbf{r}_{a} ; \varepsilon\right)$ in Eq. (13). 


\section{METAMAGNETIC TRANSITION IN FERH}

To examine the compositional sensitivity of the F-AF transition to disorder, we apply the theory to two magnetic states of a $\mathrm{Fe}_{1-x} \mathrm{Rh}_{x}-\mathrm{Rh}_{1-y} \mathrm{Fe}_{y}$ alloy at many temperatures, both with and without an external field $\vec{H}=H \hat{z}$.

(i) The first state is a ferromagnetic state $(F)$ with local moments on the Fe sites all set to $\vec{m}_{a}=m_{f} \hat{z}$, describing how aligned the Fe moments are on the Fe-rich sublattice $(A)$, and all $\vec{m}_{b}=m_{f^{\prime}} \hat{z}$, describing the analogous order parameter for the antisite Fe moments on the Rh-rich sublattice $(B)$.

(ii) The second is a canted antiferromagnetic state (AF) [23], with order parameters $\vec{m}_{a}$ alternating between $m_{f} \hat{z}+$ $m_{a f} \hat{x}$ and $m_{f} \hat{z}-m_{a f} \hat{x}$ on the two interleaved fcc sublattices which form the $A$ sublattice, and all $\vec{m}_{b}=m_{f^{\prime}} \hat{z}$ for the sites of the $B$ sublattice.

The paramagnetic state is specified by $m_{f}=m_{f^{\prime}}=m_{a f}=$ 0 , and the $T=0 \mathrm{~K}$ magnetic ground states by $m_{f}=m_{f^{\prime}}=1$, $m_{a f}=0$ for the F state and $m_{a f}=1, m_{f}=m_{f^{\prime}}=0$ for the AF state. In general $m_{f}, m_{f^{\prime}}$ are ferromagnetic order parameters while $m_{a f}$ describes the extent of antiferromagnetic order.

For specific concentrations $(x, y) \Leftrightarrow\left(c_{A}, c_{B}\right)$, and magnetic state (F or AF) we select many values of $m_{f}, m_{f^{\prime}}, m_{a f}(80-120$ sets) and calculate ab initio $[34,38] \bar{\Omega}$ and $\bar{S}_{\text {elec }}$ averaged with the probability distributions consistent with these choices. We find that in terms of $m_{f}, m_{f^{\prime}}$, and $m_{a f}$

$$
\begin{aligned}
\bar{\Omega} \approx & E_{0}-\frac{\pi^{2}}{6}\left(k_{\mathrm{B}} T\right)^{2} \bar{n}\left(E_{\mathrm{F}}\right)-c_{A}\left(e_{a f} m_{a f}^{2}+g_{a f} m_{a f}^{4}\right. \\
& \left.+e_{f} m_{f}^{2}+g_{f} m_{f}^{4}+g_{a f f} m_{a f}^{2} m_{f}^{2}\right) \\
& -c_{B}\left(e_{f f^{\prime}} m_{f} m_{f^{\prime}}+g_{a f f^{\prime}} m_{a f}^{2} m_{f^{\prime}}^{2}+g_{f f^{\prime}} m_{f}^{3} m_{f^{\prime}}\right)
\end{aligned}
$$

and

$$
\bar{n}\left(E_{\mathrm{F}}\right) \approx n_{0}+c_{A}\left(n_{a f} m_{a f}^{2}+n_{f} m_{f}^{2}\right)+c_{B} n_{f f^{\prime}} m_{f} m_{f^{\prime}}
$$

fit our ab initio computational data very well. Equation (16) reflects how the spin-polarized electronic structure adapts to the extent and type of long-range magnetic order. All fit coefficients depend on $c_{A}, c_{B}$, and the magnetic state. For the F state, $e_{a f}, g_{a f}, g_{a f f}, g_{a f f^{\prime}}$, and $n_{a f}$ coefficients are all zero and if $c_{B}=0$, when there are no Fe atoms on sublattice $B$, those coefficients associated with the $B$ sublattice are not needed. The $e_{f}$ coefficient includes the effect derived from the spin polarization on the $\mathrm{Rh}$ sites which is caused by the $\mathrm{Fe}$ local moments lining up $\left(m_{f}\right)$ [41,42].

We use Eqs. (15) and (16) in Eq. (5) to specify the free-energy function, $\mathcal{F}\left(m_{a f}, m_{f}, m_{f^{\prime}} ; c_{A}, c_{B}, \vec{H}, T\right)$, and find, for both $\mathrm{F}$ and AF states, those values of $m_{a f}, m_{f}$, and $m_{f^{\prime}}$ which minimize it for selected $T, \vec{H}, c_{A}$, and $c_{B}$ values, i.e., $\overline{\mathcal{F}}_{F}\left(\vec{H}, c_{A}, c_{B}, T\right)$ and $\overline{\mathcal{F}}_{A F}\left(\vec{H}, c_{A}, c_{B}, T\right)$. By comparing $\overline{\mathcal{F}}_{F}$ and $\overline{\mathcal{F}}_{A F}$ we can locate the F-AF transition, its magnetic field dependence, and associated MCE and electronic structure changes.

\section{A. Stoichiometric, completely B2-ordered FeRh}

Our first application is to completely ordered Fe-Rh $(x=$ $y=0$, i.e., $c_{A}=1, c_{B}=0$ ), with the lattice spacing $3.0 \AA$, in line with experiment. A local moment of $3.15 \mu_{\mathrm{B}}$ forms on the Fe atoms and a net spin polarization is induced on $\mathrm{Rh}$ of $1.00 \mu_{\mathrm{B}}$ per atom, when the Fe moments are fully ferromagnetically aligned $\left(m_{f}=1\right)$ but vanishes when the Fe local moments are disordered $\left(m_{f}=0\right)$. The coefficients in Eqs. (15) and (16) are calculated to be (in meV) $e_{a f}=87.6, e_{f}=99.4, g_{a f}=18.2$, $g_{f}=-23.0$, and $g_{a f f}=-35.6 . e_{f}>e_{a f}$ so that in zero field the material orders ferromagnetically at $T_{c}=773 \mathrm{~K}$ while the negative signs of $g_{f}$ and $g_{a f f}$ ensure a transition to an AF state at $T_{t}=495 \mathrm{~K}$ (experimental values are $T_{c}=670 \mathrm{~K}$ and $\left.T_{t}=340 \mathrm{~K}\right)$. There is a large change to $\bar{n}\left(E_{\mathrm{F}}\right)$ at $T_{t}$ (i.e., change of $\left(n_{a f} m_{a f}^{2}+n_{f} m_{f}^{2}\right)$ [9] with $n_{a f}=-1.3$ and $n_{f}=0.59$ states/eV/FeRh pair) where the order parameters are $m_{f}=0.59, m_{a f}=0$ above $T_{t}$ and $m_{f}=0, m_{a f}=0.71$ below.

We include the effects of an applied field $\vec{H}$ and find $\frac{d T_{t}}{d H}=-3.7 \mathrm{~K} \mathrm{~T}^{-1}$. The isothermal entropy change for $2 \mathrm{~T}$ has a maximum value at the transition of $|\Delta S|=21.1 \mathrm{~J} \mathrm{~K}^{-1} \mathrm{~kg}^{-1}$ which compares well with values reported experimentally $[4,16,43]$ of $|\Delta S|=13-20 \mathrm{~J} \mathrm{~K}^{-1} \mathrm{~kg}^{-1}$. Notably $|\Delta S|$ has a large electronic component-reducing to $13 \mathrm{~J} \mathrm{~K}^{-1} \mathrm{~kg}^{-1}$ if $\bar{S}_{\text {elec }}=\frac{\pi^{2}}{3} k_{B}^{2} T \bar{n}\left(E_{\mathrm{F}}\right)$ [see Eq. (15)] is omitted.

Figure 1(a) shows the sublattice-resolved [53] electronic structure for $\mathrm{FeRh}$ above $T_{c}\left(m_{f}=0, m_{a f}=0\right)$. In the paramagnetic state, the $\mathrm{Fe}$ atoms still retain a local exchange splitting [30] that underpins the stability of the disordered local moments although, once averaged over all the orientations equally weighted, there is no net spin polarization in the system. In a F state, however, $m_{f} \neq 0$ and the Rh-related states become spin polarized by the overall long-range alignment of the Fe local moments. This lowers the bonding states slightly, as shown in Fig. 2(a) for $T$ just above $T_{t}$, in comparison with what happens when the Rh states are unpolarized in an AF state $\left(m_{a f} \neq 0\right)$ as shown in Fig. 2(b) for $T$ just below $T_{t}$. For the $\mathrm{F}$ state there is a DOS peak $1.6 \mathrm{eV}$ below $E_{\mathrm{F}}$ which is slightly lower than its AF counterpart and one set of higher-lying states is mostly filled for majority spin electrons as seen in the double peak structure straddling $E_{\mathrm{F}}$ at $\pm 0.3 \mathrm{eV}$. This energy gain is overturned for more entrenched AF order, also evident in Fig. 2(b). For large $m_{a f}$, the AF coupling between Fe local moments causes a redistribution of states so that a rather pronounced trough develops in the DOS at $E_{\mathrm{F}}$. The differing effect of $\mathrm{F}$ and $\mathrm{AF}$ order on this redistribution $[5,10,54]$ generates the large electronic MCE component and underpins the transition.

\section{B. Off stoichiometry and incomplete B2-order: The effect of $\mathrm{Fe}$ "anti-site" defects}

Our most important result is the profound effect that tiny compositional changes have on this electronic structure balancing act. When we model slightly incomplete B2 order by swapping just $2 \%$ of Fe with $\mathrm{Rh}(x=y=0.02)$ we find $T_{t}$ to plummet to $208 \mathrm{~K}$ as found experimentally [12] and $T_{c}$ to increase to $859 \mathrm{~K}$. For a $4 \%$ swap the F-AF transition vanishes completely $\left(T_{c}=926 \mathrm{~K}\right)$. Shifting the composition off stoichiometric has dramatic consequences too. No F-AF transition is found for the Fe-rich $x=0, y=0.04$ $\mathrm{Fe}_{52} \mathrm{Rh}_{48}$ composition $\left(T_{c}=1008 \mathrm{~K}\right)$ while for $x=0.04$, $y=0\left(\mathrm{Fe}_{48} \mathrm{Rh}_{52}\right) T_{t}$ increases to $549 \mathrm{~K}\left(T_{c}=700 \mathrm{~K}\right)$. For the $\mathrm{Fe}_{49} \mathrm{Rh}_{51}$ composition with slightly imperfect B2 order, consistent with reported annealing temperatures $(x=0.03$, 

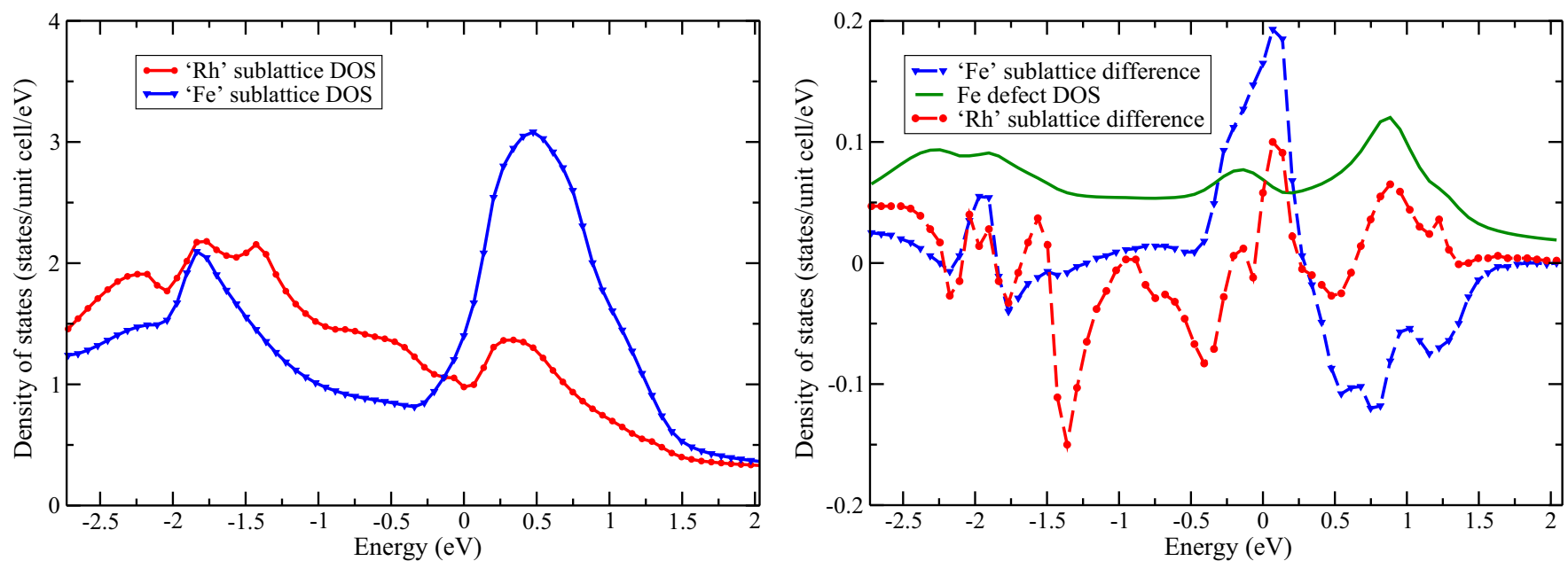

FIG. 1. (Color online) (a) Density of states of Fe-Rh above $T_{c}$ resolved into sublattice components. (b) The difference in the DOS between Fe-rich $\mathrm{Fe}-\mathrm{Rh}_{0.96} \mathrm{Fe}_{0.04}$ and $\mathrm{Fe}-\mathrm{Rh}$ above $T_{c}$. The Fe "defect" DOS on the Rh sublattice is also shown.

$y=0.01)$, we find $T_{t}=415 \mathrm{~K}\left(T_{c}=815 \mathrm{~K}\right)$ and $\left|\Delta S^{\max }\right|=$ $20.7 \mathrm{~J} \mathrm{~K}^{-1} \mathrm{Kg}^{-1}$ at $2 \mathrm{~T}$, in good agreement with experiment [4].

The transition's extreme sensitivity to Fe defects on the $\mathrm{Rh}$ sublattice is encapsulated by the relatively large positive value of the $e_{f f^{\prime}}$ coefficient in Eq. (15) which describes the ferromagnetic coupling between $\mathrm{Fe}$ atoms on the two sublattices $(\approx 600 \mathrm{meV})$. Figure $1(\mathrm{~b})$ shows the root electronic cause for this. With the presence of local spin polarization on the Fe "defect" sites on the Rh sublattice, developing F order deepens the DOS trough at $E_{\mathrm{F}}$ with near depletion of a set of minority spin states. $T=0 \mathrm{~K}$ DFT supercell calculations of the band structure of FM FeRh with Fe antisite defects [55] support this interpretation. This enhances the material's tendency to F order and the F-AF transition is affected accordingly. Fe antisite defects reduce the electronic contribution to the MCE, e.g., for $x=y=0.02$, only $20 \%$ of the $|\Delta S|=22.3 \mathrm{~J} \mathrm{~K}^{-1} \mathrm{~kg}^{-1}$ is electronic. So magnetotransport properties are also acutely composition sensitive in this material.

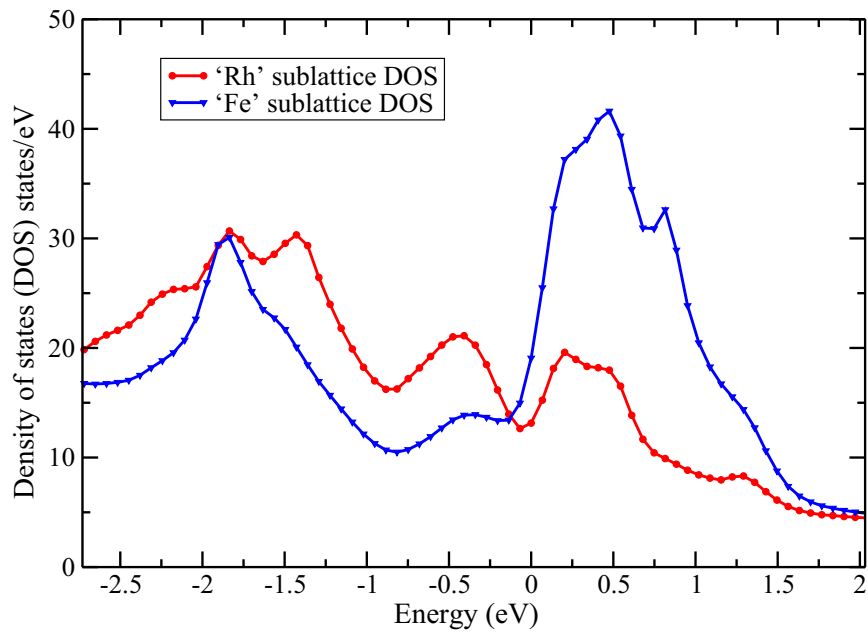

\section{Impurity-doping and volume effects}

The metamagnetism of Fe-Rh varies with impurity doping [17] and this can also be linked to the Fe antisite effect. For example isoelectronic dopants such as $\mathrm{Pd}$ and Pt have opposite effects on $T_{t}$ owing the differing propensities for the dopants to displace Fe atoms onto the $\mathrm{Rh}$ sublattice. $T=0 \mathrm{~K}$ calculations [47] for the energy difference between $\mathrm{F} \mathrm{Fe}_{1-2 z} \mathrm{Pt}_{2 z}-\mathrm{Rh}_{1-z} \mathrm{Fe}_{z}$ and $\left.\mathrm{Fe}_{1-z} \mathrm{Rh}_{z}-\mathrm{Rh}_{1-2 z} \mathrm{Pt}_{2 z}\right)$ alloys $(0<z<10 \%)$ show that the "big" Pt atoms preferentially displace $\mathrm{Rh}$ atoms to the $\mathrm{Fe}$ sublattice so that they maximize the number of the smaller Fe nearest neighbors [56]. This reduces the spin-polarization energy gain, strengthening AF ordering and increasing $T_{t}$. On the other hand similar calculations for the Pd-doped alloys show that $\mathrm{Fe}$ and $\mathrm{Rh}$ atoms are displaced roughly equally so that some Fe atoms find their way onto the Rh sublattice creating Fe antisite defects. Consequently $T_{t}$ drops [17].

At the F-AF transition there is a significant well-studied volume magnetostriction [57] $\lambda$ of $\approx 8 \times 10^{-3}$. We find the leading coefficients $e_{f}$ and $e_{a f}$ of Eq. (15) to change by 16 and $-11 \mathrm{meV}$ respectively for a $1 \%$ volume increase reflecting

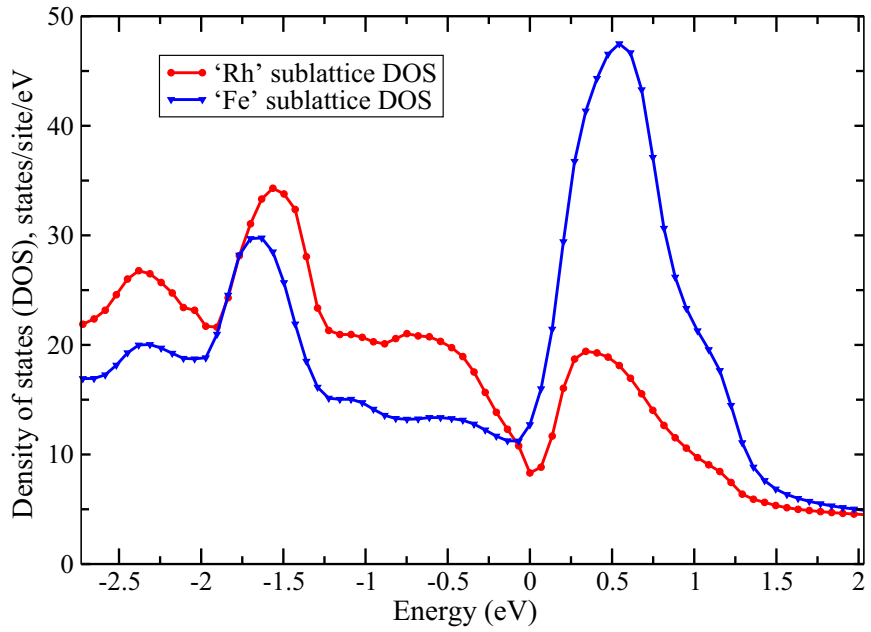

FIG. 2. (Color online) (a) Density of states of FeRh just above $T_{t}$ in the F state, $m_{f}=0.59$, and (b) just below $T_{t}$ in the AF state, $m_{a f}=0.71$. 
the increasing tendency towards $\mathrm{F}$ order with expansion. So the free energy $\mathcal{F} \approx \mathcal{F}_{0}+\mathcal{F}^{\prime} \lambda+\frac{1}{2} K \lambda^{2}$. Using a bulk modulus $K$ estimate of 2 Mbar [24], $\lambda$ at $T_{t}$ is $4 \times 10^{-3}$ for perfectly ordered Fe-Rh and $6 \times 10^{-3}$ for imperfectly ordered $\mathrm{Fe}_{98} \mathrm{Rh}_{2}-\mathrm{Rh}_{98} \mathrm{Fe}_{2}$ alloy, in fair agreement with experiment, and we deduce that volume changes are consequences of the magnetic transition but not major drivers of it.

\section{CONCLUSIONS}

We have described an ab initio DFT-based theory for magnetic materials which gives a quantitative account of a material's properties as function of temperature and applied magnetic field. The role of temperature-dependent spinpolarized electronic structure is paramount. The unusual ordered FeRh material with its famous metamagnetic transition has all main features described well in comparison with experiment:

(i) the location of the F-AF transition close to room temperature,

(ii) the magnitude of the magnetocaloric entropy changes with large electronic contributions, and

(iii) crucially the extremely narrow compositional range for the first-order transition and its dependence on material preparation route.
In particular we show why very small variations away from complete B2 order and stoichiometry have such a strong effect on the transition in terms of the presence of Fe antisites, i.e., $\mathrm{Fe}$ atoms occupying a very small proportion of the Rh sublattice sites. In addition to affecting phase coexistence and the broadening of the first-order magnetic transition, this compositional hypersensitivity means that, even if $\mathrm{Rh}$ were cheaply available, FeRh is unlikely to become a technologically useful adaptive magnetic material. On the other hand the theory's facility to quantify subtle compositional and electronic effects means that it can aid in the design of the next generation of adaptive magnetic materials including magnetic refrigerants based on, for example, MnFePSi or LaFeSi [43].

\section{ACKNOWLEDGMENTS}

Support is acknowledged from EPSRC (UK) Grant No. EP/J006750/1 (J.B.S. and R.B.), the HGF-YIG Programme VH-NG-717 (Functional Nanoscale Structure and Probe Simulation Laboratory) (M.S.D.), and the European Union and the State of Hungary, co-financed by the European Social Fund, in the framework of TÁMOP-4.2.4.A/1-11/1-20120001 National Excellence Program (A.D.).
[1] Y. Imry and M. Wortis, Phys. Rev. B 19, 3580 (1979).

[2] S. B. Roy, Acta Mater. 56, 5895 (2008).

[3] M. Fallot, Ann. Phys. (Leipzig) 10, 291 (1938).

[4] M. P. Annaorazov, S. A. Nikitin, A. L. Tyurin, K. A. Asatryan, and A. Kh. Dovletov, J. Appl. Phys. 79, 1689 (1996).

[5] J. van Driel, R. Coehoorn, G. J. Strijkers, E. Brück, and F. R. de Boer, J. Appl. Phys. 85, 1026 (1999).

[6] Y. Kobayashi, K. Muta, and K. Asai, J. Phys.: Condens. Matter 13, 3335 (2001).

[7] S. Maat, J.-U. Thiele, and E. E. Fullerton, Phys. Rev. B 72, $214432(2005)$.

[8] C. Stamm, J.-U. Thiele, T. Kachel, I. Radu, P. Ramm, M. Kosuth, J. Minar, H. Ebert, H. A. Dürr, W. Eberhardt, and C. H. Back, Phys. Rev. B 77, 184401 (2008).

[9] I. Suzuki, T. Naito, M. Itoh, T. Sato, and T. Taniyama, J. Appl. Phys. 109, 07C717 (2011).

[10] M. A. de Vries, M. Loving, A. P. Mihai, L. H. Lewis, D. Heiman, and C. H. Marrows, New J. Phys. 15, 013008 (2013).

[11] J. S. Kouvel, J. Appl. Phys. 37, 1257 (1966).

[12] P. Tu, A. J. Heeger, J. S. Kouvel, and J. B. Comly, J. Appl. Phys. 40, 1368 (1969).

[13] S. Hashi, S. Yanase, Y. Okazaki, and M. Inoue, IEEE Trans. Magn. 40, 2784 (2004).

[14] J. W. Cao, N. T. Nam, S. Inoue, H. Y. Y. Ko, N. N. Phuoc, and T. Suzuki, J. Appl. Phys. 103, 07F501 (2008).

[15] K. Aikoh S. Kosugi, T. Matsui, and A. Iwase, J. Appl. Phys. 109, $07 \mathrm{E} 311$ (2011).

[16] D. W. Cooke, F. Hellman, C. Baldasseroni, C. Bordel, S. Moyerman, and E. E. Fullerton, Phys. Rev. Lett. 109, 255901 (2012).

[17] R. Barua, F. Jimenez-Villacorta, and L. H. Lewis, Appl. Phys. Lett. 103, 102407 (2013).
[18] M. P. Annaorazov, K. A. Asatryan, G. Myalikgulyev, S. A. Nikitin, A. M. Tishin, and A. L. Tyurinet, Cryogenics 32, 867 (1992).

[19] J. Thiele, S. Maat, and E. E. Fullerton, Appl. Phys. Lett. 82, 2859 (2003).

[20] G. Ju, J. Hohlfeld, B. Bergman, R. J. M. van de Veerdonk, O. N. Mryasov, J-Y. Kim, X. Wu, D. Weller, and B. Koopmans, Phys. Rev. Lett. 93, 197403 (2004).

[21] L. M. Sandratskii and P. Mavropoulos, Phys. Rev. B 83, 174408 (2011).

[22] S. O. Mariager, F. Pressacco, G. Ingold, A. Caviezel, E. MöhrVorobeva, P. Beaud, S. L. Johnson, C. J. Milne, E. Mancini, S. Moyerman, E. E. Fullerton, R. Feidenhansl, C. H. Back, and C. Quitmann, Phys. Rev. Lett. 108, 087201 (2012).

[23] P. M. Derlet, Phys. Rev. B 85, 174431 (2012).

[24] V. L. Moruzzi and P. M. Marcus, Phys. Rev. B 46, 2864 (1992).

[25] M. E. Gruner, E. Hoffmann, and P. Entel, Phys. Rev. B 67, 064415 (2003).

[26] O. N. Mryasov, Phase Transit. 78, 197 (2005).

[27] R. Y. Gu and V. P. Antropov, Phys. Rev. B 72, 012403 (2005).

[28] J. Kübler, Theory of Itinerant Electron Magnetism (Clarendon, Oxford, 2000).

[29] W. Bragg and E. Williams, Proc. R. Soc. A 145, 699 (1934).

[30] B. L. Gyorffy, A. J. Pindor, J. Staunton, G. M. Stocks, and H. Winter, J. Phys. F 15, 1337 (1985).

[31] J. Hubbard, Phys. Rev. B 20, 4584 (1979).

[32] H. Hasegawa, J. Phys. Soc. Jpn. 46, 1504 (1979).

[33] J. B. Staunton and B. L. Gyorffy, Phys. Rev. Lett. 69, 371 (1992).

[34] J. B. Staunton, S. Ostanin, S. S. A. Razee, B. L. Gyorffy, L. Szunyogh, B. Ginatempo, and E. Bruno, Phys. Rev. Lett. 93, 257204 (2004). 
[35] M. dos Santos Dias, J. B. Staunton, A. Deak, and L. Szunyogh, Phys. Rev. B 83, 054435 (2011).

[36] I. D. Hughes, M. Däne, A. Ernst, W. Hergert, M. Lüders, J. Poulter, J. B. Staunton, A. Svane, Z. Szotek, and W. M. Temmerman, Nature (London) 446, 650 (2007).

[37] I. D. Hughes, M. Däne, A. Ernst, W. Hergert, M. Lüders, J. B. Staunton, Z. Szotek, and W. M. Temmerman, New J. Phys. 10, 063010 (2008).

[38] J. B. Staunton, M. dos Santos Dias, J. Peace, Z. Gercsi, and K. G. Sandeman, Phys. Rev. B 87, 060404(R) (2013).

[39] J. B. Staunton, L. Szunyogh, A. Buruzs, B. L. Gyorffy, S. Ostanin, and L. Udvardi, Phys. Rev. B 74, 144411 (2006).

[40] R. P. Feynman, Phys. Rev. 97, 660 (1955).

[41] M. Lezaic, P. Mavropoulos, G. Bihlmayer, and S. Blügel, Phys. Rev. B 88, 134403 (2013).

[42] A. Deak, E. Simon, L. Balogh, L. Szunyogh, M. dos Santos Dias, and J. B. Staunton (unpublished).

[43] K. G. Sandeman, Scr. Mater. 67, 566 (2012).

[44] N. D. Mermin, Phys. Rev. 137, A1441 (1965).

[45] G. M. Stocks, W. M. Temmerman, and B. L. Gyorffy, Phys. Rev. Lett. 41, 339 (1978).

[46] D. D. Johnson, D. M. Nicholson, F. J. Pinski, B. L. Gyorffy, and G. M. Stocks, Phys. Rev. Lett. 56, 2088 (1986).
[47] H. Ebert, D. Ködderitzsch, and J. Minar, Rep. Prog. Phys. 74, 096501 (2011).

[48] P. Strange, J. Staunton, and B. L. Gyorffy, J. Phys. C 17, 3355 (1984).

[49] J. P. Perdew and Y. Wang, Phys. Rev. B 45, 13244 (1992).

[50] J. Harris, Phys. Rev. B 31, 1770 (1985).

[51] J. S. Faulkner and G. M. Stocks, Phys. Rev. B 21, 3222 (1980).

[52] D. Ködderitzsch, S. Lowitzer, J. B. Staunton, and H. Ebert, Phys. Status Solidi B 248, 2248 (2011).

[53] C. Bordel, J. Juraszek, D. W. Cooke, C. Baldasseroni, S. Mankovsky, J. Minar, H. Ebert, S. Moyerman, E. E. Fullerton, and F. Hellman, Phys. Rev. Lett. 109, 117201 (2012).

[54] A. X. Gray, D. W. Cooke, P. Krüger, C. Bordel, A. M. Kaiser, S. Moyerman, E. E. Fullerton, S. Ueda, Y. Yamashita, A. Gloskovskii, C. M. Schneider, W. Drube, K. Kobayashi, F. Hellman, and C. S. Fadley, Phys. Rev. Lett. 108, 257208 (2012).

[55] Y. Kaneta, S. Ishino, Y. Chen, S. Iwata, and A. Iwase, Jpn. J. Appl. Phys. 50, 105803 (2011).

[56] F. J. Pinski, B. Ginatempo, D. D. Johnson, J. B. Staunton, G. M Stocks, and B. L. Gyorffy, Phys. Rev. Lett. 66, 766 (1991).

[57] J. B. McKinnon, D. Melville, and E. W. Lee, J. Phys. C 3, S46 (1970). 